\title{
PHOTOLUMINESCENCE PROPERTIES OF POROUS SILICON PREPARED BY ELECTROCHEMICAL ETCHING OF Si EPITAXIAL LAYER
}

\author{
E. Nossarzewska-Orlowska and A. Brzozowski
}

Institute of Electronic Materials Technology, Wólczyńska 133, 01-919 Warszawa, Poland

\begin{abstract}
The photoluminescence properties of porous layers prepared by anodization of $p / p^{+}$silicon epitaxial wafers are presented. The shift of the photoluminescence spectrum towards shorter wavelength due to the porosity increase and the experimental dependence of the photoluminescence maximum position on HF concentration during anodization are shown. Degradation of the photoluminescence intensity dependence on the storage time is described.

PACS numbers: 78.55.Hx, 81.60.-j
\end{abstract}

The observation made by Canham [1] of photoluminescence phenomena in porous silicon has led to new studies of this material as it may have promising applications in Si-based optoelectronic integrated circuits and displays.

The luminescence is often interpreted as resulting from the quantum confinement in small size crystallites of porous Si [1-4]. The quantum size effect leads to an increase in the band gap of the crystallites compared to the bulk $\mathrm{Si}$, which could explain the light emission in the visible range. Other models based on the luminescence properties of polysilanes [5] and siloxene [6] have been also proposed.

Porous Si layers are produced by the anodic dissolution of single-crystalline Si wafers in hydrofluoric acid (HF) solution. In our experiments we have used the 3 inches epitaxial wafers consisting of boron doped substrate with the resistivity of $0.01-0.02 \Omega \mathrm{cm}$ and $15 \mu \mathrm{m}$ thick boron doped epitaxial layer with the resistivity of $10 \Omega \mathrm{cm}$, deposited in the standard chemical vapour deposition (CVD) process. As the substrate was of low resistivity, no additional procedure such as an ion implantation or a metal deposition on the back side of the wafer was needed. The current density was kept $10 \mathrm{~mA} / \mathrm{cm}^{2}$. To obtain the layers of different porosity the etching processes were performed in the HF-isopropyl alcohol solutions with the $\mathrm{HF}$ concentration ranging from $40 \mathrm{wt} \%$ to $10 \mathrm{wt} \%$. The decrease in the $\mathrm{HF}$ concentration caused an increase in the layer porosity. The porosity was estimated by the standard gravimetric method.

For the photoluminescence measurements a conventional setup was used including the SPM-2 monochromator and the S1 cathode photomultiplier in conjunction with lock-in technique. 


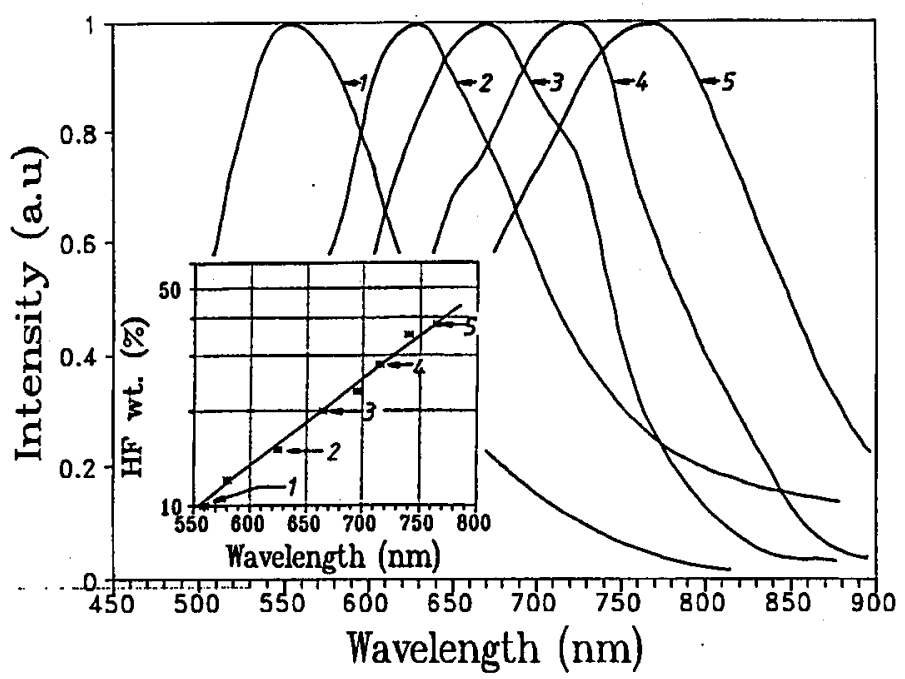

Fig. 1. Dependence of PL peak position on porous Si porosity: $1-85 \% ; 2-80 \%$; $3-75 \% ; 4-66 \% ; 5-55 \%$; spectrum 1 - excitation by UV radiation $(\lambda=350 \mathrm{~nm})$; spectra $2-5$ - excitation by $488 \mathrm{~nm}$ line of $\mathrm{Ar}^{+}$laser. In the inset: PL peak position vs. $\mathrm{HF}$ concentration.

Figure 1 shows the normalized photoluminescence spectra (PL) from the porous layers formed in the electrolyte of different HF concentration. The presented spectra show the shift of the maximum emission towards shorter wavelengths as the porosity increases. The observed "blue shift" is in agreement with the quantum size effect model proposed by Canham [1]. The spectra exhibit some additional maxima and shoulders. The full width at half maximum (FWHM) of the PL bands changes from $190 \mathrm{~nm}$ to $140 \mathrm{~nm}$ going down with a wavelength. A broad PL band, a structural character of spectrum and the continuous shift of the maximum emission by changing the etching conditions suggest the existence of various optical transition processes, possibly because of a dispersion of the Si crystallites size.

The PL of porous Si prepared by the electrochemical dissolution suffers from the problems of instability and degradation. The PL intensity decreases during air storage, as well as under excitation. Studying degradation effects in our samples we have found that:

- during storage in the air the red PL dropped to about 50\% of as-prepared sample in the period of one year. In the stored samples "a blue shift" of about $40 \mathrm{~nm}$ was observed as a result of oxidation of the Si skeleton;

- under $\mathrm{Ar}^{+}$laser excitation the PL degradation depended on the selected laser line (faster for $514 \mathrm{~nm}$ than for $488 \mathrm{~nm}$ ) and on the incident flux.

Degradation depends critically on the sample storage time (Fig. 2). In the as-prepared samples we have observed the increase in the PL intensity under laser illumination during the first few days even using high laser beam power density $\left(1000 \mathrm{~mW} / \mathrm{cm}^{2}\right)$. After this period the decrease in PL intensity has occurred under 


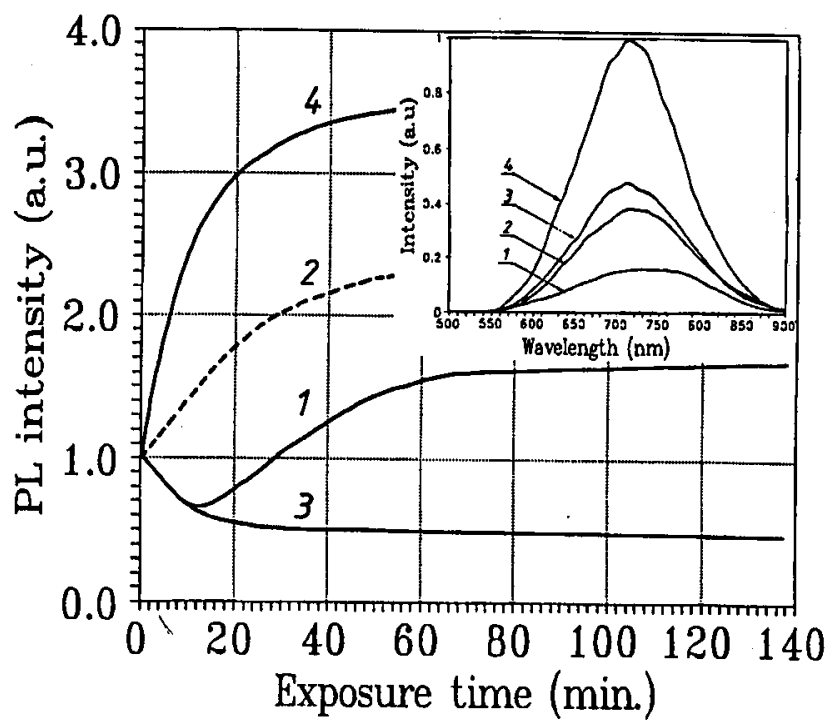

Fig. 2. Change of the PL intensity at $\lambda=750 \mathrm{~nm}$ under illumination by $\mathrm{Ar}^{+}$laser $488 \mathrm{~nm}$ line vs. exposure time. 1 - as-prepared sample; 2,3 - after two week storage; 4 - after six month storage and 1 hour treatment in $\mathrm{H}_{2} \mathrm{O}_{2}$. Laser beam power density: $1,3,4-1000 \mathrm{~mW} / \mathrm{cm}^{2} ; 2-1 \mathrm{~mW} / \mathrm{cm}^{2}$. In the inset: enhancement effect of a treatment in $\mathrm{H}_{2} \mathrm{O}_{2}$ on the PL intensity. 1 - sample before treatment; 2-4 - samples treated in $\mathrm{H}_{2} \mathrm{O}_{2}$ for: $2-10 \mathrm{~min}, 3-30 \mathrm{~min}, 4-90 \mathrm{~min}$. Laser beam power density $1000 \mathrm{~mW} / \mathrm{cm}^{2}$.

high power beam but attenuation of the flux by neutral filters has still caused the increase in the PL intensity. After long storage in the air (a few months) even a low excitation flux causes the degradation of the PL intensity. In the next experiment we have immersed our long stored samples in a $30 \% \mathrm{H}_{2} \mathrm{O}_{2}$ solution. The PL intensity at $\lambda_{\max }$ increased about 8 times after one hour treatment and we have observed again the increase in the intensity under laser excitation.

From our results we conclude that surface passivation, either by $\mathrm{Si}-\mathrm{H}_{x}$ species formed during etching in $\mathrm{HF}$ or by certain $\mathrm{Si}-\mathrm{O}-\mathrm{H}$ and $\mathrm{Si}-\mathrm{O}$ bonds formed in a presence of oxidizing agent $\left(\mathrm{H}_{2} \mathrm{O}_{2}\right.$ in our experiment), as well as under laser irradiation on $\mathrm{H}$ covered surface, results in an increased PL.

\section{References}

[1] L.T. Canham, Appl. Phys. Lett. 57, 1046 (1990).

[2] V. Lehmann, U. Gosele, Appl. Phys. Lett. 58, 856 (1991).

[3] A.J. Read, R.J. Needs, K.J. Nash, L.T. Canham, P.D.J. Callcot, A. Qteish, Phys. Rev. Lett. 69, 1232 (1992).

[4] I. Sagnes, A. Halimaoui, G. Vincent, P.A. Badoz, Appl. Phys. Lett. 62, 1156 (1993). 
[5] S.M. Prokes, J. Appl. Phys. 74, 407 (1993).

[6] M.S. Brandt, H.D. Fuchs, M. Stutzmann, J. Weber, M. Cardona, Solid State Commun. 81, 307 (1992). 